\title{
Columnar dimer and plaquette resonating-valence-bond orders in the quantum dimer model
}

\author{
P. W. Leung* and K. C. Chiu \\ Department of Physics, Hong Kong University of Science and Technology, Clear Water Bay, Hong Kong \\ Karl J. Runge \\ Department of Physics, Lawrence Livermore National Laboratory, University of California, Livermore, California 94551
}

(Received 28 May 1996)

\begin{abstract}
We study the nature of the ground state of the quantum dimer model proposed by Rokhsar and Kivelson by diagonalizing the Hamiltonian of the model on square lattices of size $L \times L$, where $L \leqslant 8$, with periodic boundary conditions. Finite-size scaling studies of the columnar order parameter and the low lying excitation spectrum show no evidence of a dimer liquid state in any finite region of the zero temperature phase diagram. In addition, we find evidence of a transition from the columnar dimer state to an intermediate state at a negative value of $V / J$. This state is consistent with the plaquette resonating-valence-bond state. The energy gap of this state vanishes as a power law of $L$. It exhibits columnar dimer order, but has disorder within the dimer columns. This state persists up to $V / J<1$, and the system changes to a dimer liquid state only at $V / J=1$. [S0163-1829(96)08242-2]
\end{abstract}

\section{INTRODUCTION}

The quantum dimer model (QDM) was first introduced by Rokhsar and Kivelson (RK). ${ }^{1}$ It was proposed as an alternative description of the non-Néel state of the spin- $\frac{1}{2}$ Heisenberg antiferromagnet on a square lattice. In a state with exponentially decaying spin-spin correlation and a large energy gap separating the spin excitations from the singlet ground state, RK argued that the low energy physics is contained in the "short-range resonating valence bond (RVB)", states, spanned by the set of nearest-neighbor valence bond states. Nearest-neighbor valence bonds are called dimers. The basis set thus consists of all possible dimer configurations at closest-packing (all sites form exactly one dimer with one of their nearest neighbors). RK also argued that the nonorthogonality of these basis states can be absorbed by defining a phenomenological Hamiltonian with short range dimer interactions. The relevance of this quantum dimer model to the frustrated quantum antiferromagnet was supported by other independent studies. Large- $N$ expansion, ${ }^{2,3}$ series expansion, ${ }^{4}$ and numerical diagonalization ${ }^{5}$ have shown that a spin-Peierls state exists in some region of the phase diagram where the Néel state is unstable. This spin-Peierls state corresponds to the state in the QDM where the dimers are frozen into a columnar pattern [Fig. 1(a)]. Recently the QDM has been generalized to the kagomé lattice by Zeng and Elser. ${ }^{6}$

The Hamiltonian of the QDM proposed by RK is

$$
\mathcal{H}=\sum_{\text {plaquettes }}[-J(|\|\rangle\langle=|+\text { H.c. })+V(|=\rangle\langle=|+| \|\rangle\langle\||)] .
$$

$\|$ and $=$ represent parallel dimers on the opposite sides of a square plaquette. The first term of $\mathcal{H}$ is the dimer kinetic energy operator while the second term is the potential energy. The space spanned by all close-packed dimer configurations on a square lattice can be divided into distinct topo- logical sections characterized by a pair of conserved winding numbers $\left(\Omega_{x}, \Omega_{y}\right)$ (refer to Appendix A). On an $L \times L$ square lattice, the allowed winding numbers are $-L / 2 \leqslant \Omega_{x}, \Omega_{y} \leqslant L / 2$. At $V / J>1$, the exact ground state of the QDM is the staggered dimer solid shown in Fig. 1(b). It is fourfold degenerate and has winding numbers $( \pm L / 2,0)$ and $(0, \pm L / 2)$. At $V / J \ll-1$, the ground state develops columnar order as shown in Fig. 1(a). At $V / J=1$, the lowest energy states in all topological sectors are zero-energy eigenstates of $\mathcal{H}$. They are equal superpositions of all the dimer configurations in their sectors. Therefore, the ground state at $V / J=1$ is a dimer liquid, and any ground state dimer correlation function can be calculated exactly by the method of Fisher and Stephenson ${ }^{7}$ for the classical dimer problem. Consequently this state is called the FS state. The ground state properties of the QDM in the range $0 \leqslant V / J<1$ are not so clear. There are three main questions: (a) is the ground state in this range ordered (dimer solid) or disordered (dimer liquid)? (b) If it is ordered, what is the nature of this order? And (c) if it is disordered, is there a gap in the low lying excitation spectrum? RK suggested that a dimer liquid state can exist in a finite region of $V / J<1$. By numerically diagonalizing the QDM on square lattices up to $6 \times 6$, Sachdev $^{8}$ found evidence for the columnar state, but no evidence for a dimer liquid state at $V / J \neq 1$. However, due to finite-size effects, he did not rule out the possibility that dimer liquid exists over a finite range $\kappa<V / J \leqslant 1$, and estimated a lower

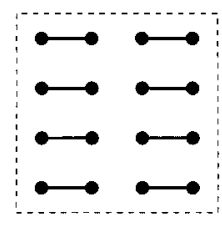

(a)

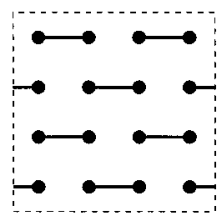

(b)

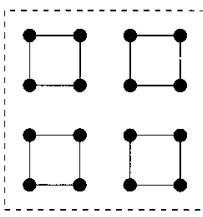

(c)
FIG. 1. The (a) columnar, (b) staggered, and (c) plaquette RVB states. 


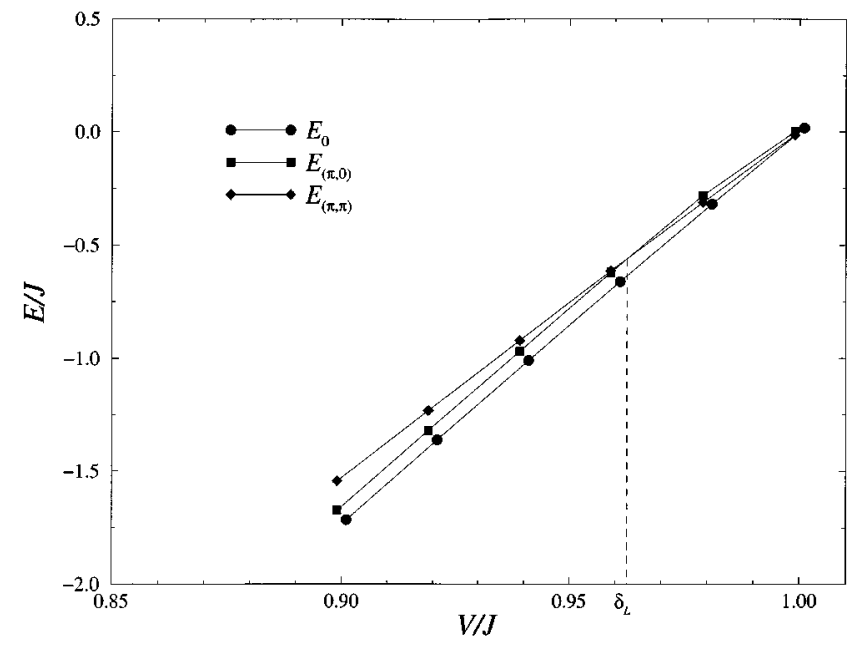

FIG. 2. Energy levels $E_{0}, E_{(\pi, 0)}$, and $E_{(\pi, \pi)}$ for the $L=8$ system. All other energy levels are higher than these three and are not shown.

bound $0.5<\kappa$. On the other hand, some analytic studies have suggested the existence of a dimer liquid state at $V / J \neq 1$. By mapping the QDM to a roughening problem, Levitov ${ }^{9}$ showed that a dimer liquid with gapless excitations may exist. Orland ${ }^{10}$ formulated the QDM as a system of noninteracting fermionic strings, and claimed that at $V / J=0$, the ground state is a dimer liquid. From this he inferred that the ground state is a dimer liquid for $0 \leqslant V / J \leqslant 1$, with a phase transition to a dimer solid at some $V / J<0$. We also note that another kind of dimer order has been proposed, ${ }^{2,11-13}$ although in a different context. This is a plaquette RVB state as shown in Fig. 1(c), where every other plaquette is in the $|\|\rangle$ or $|=\rangle$ state with equal probability and independent of other plaquettes. In this paper, we extend Sachdev's calculations to an $8 \times 8$ square lattice. We aim to identify the different phases of the QDM in the range $V / J<1$.

\section{NUMERICAL CALCULATIONS}

Using the Lanczos algorithm, we diagonalize the Hamiltonian $\mathcal{H}$ in the range $-1<V / J<1$ on an $L \times L$ square lattice with periodic boundary conditions, where $L=4,6$, and 8. The basis states are all possible dimer configurations at closest packing. The number of basis states for a given $L$ can be evaluated ${ }^{7}$ analytically. Enumerating all the dimer configurations is much more difficult. Nevertheless, we have devised an efficient algorithm which enumerates all the 300 million dimer configurations on the $L=8$ lattice within four hours using an HP 735 workstation (see Appendix B). Using translational symmetry, we can reduce the number of basis states on an $L \times L$ lattice by a factor of about $L^{2}$. For $V / J<1$, the ground state has momentum $\mathbf{k}=(0,0)$, and winding numbers $(0,0)$. By restricting the basis states to the topological sector with winding numbers $(0,0)$, the number of basis states can be further reduced by a factor of about 2 . To calculate the ground state eigenvector, we restrict ourselves to the subspace with momentum $\mathbf{k}=(0,0)$ and winding numbers $(0,0)$. The number of basis states in this subspace is about 2.4 million. This makes the numerical diagonalization possible on a workstation.

\section{SYMMETRIES OF LOW LYING EXCITED STATES}

To study the different phases in the range $-1<V / J<1$, we first study the symmetries of the low lying excited states. They are important in finite-size studies. If a system possesses a broken symmetry in the thermodynamic limit, the ground state of the finite system will still be totally symmetric. In this case the ground state expectation of the appropriate order parameter will have long-range correlations, and there will exist low lying excited states with the appropriate symmetries. The columnar dimer state shown in Fig. 1(a) has winding numbers $(0,0)$ and is fourfold degenerate. These degenerate states can be combined to form four states, two with momentum $(0,0)$, and two with momenta $(\pi, 0)$ and $(0, \pi)$. Consequently in a finite system which possesses columnar order in the thermodynamic limit, the state with momentum $(0,0)$ will be the ground state and the others will appear as low lying excited states, which are degenerate with the ground state in the thermodynamic limit. Note that the same is true for the plaquette RVB state shown in Fig. 1(c). Therefore, low lying excited states with momenta $(\pi, 0)$ and $(0, \pi)$ are consistent with the existence of columnar or plaquette RVB order in the ground state.

We calculate the energies of the lowest few eigenstates of the QDM on the $L=4,6$, and 8 lattices for $-1 \leqslant V / J<1$. All three lattices show qualitatively the same picture. The ground state $E_{0}$ always has zero momentum and winding numbers, as mentioned in Sec. II. We can identify two sets of low lying excited states: except when $V / J$ is close to 1 , the lowest set of excited states is degenerate with momenta $(\pi, 0)$, and $(0, \pi)$, and has zero winding numbers. We call it $E_{(\pi, 0)}$. In the same region, the set of next lowest excited state is degenerate with momenta $(0,0)$ and $(\pi, \pi)$, and has winding numbers $( \pm 1,0)$ and $(0, \pm 1)$. We call it $E_{(\pi, \pi)}$. In Fig. 2 we plot the energies of $E_{0}, E_{(\pi, 0)}$, and $E_{(\pi, \pi)}$ in a small range of $V / J$ close to 1 for the $L=8$ system. We note that the energy gaps of $E_{(\pi, 0)}$ and $E_{(\pi, \pi)}$ both go to zero as $V / J$ approaches 1 . In addition, there exist a $\delta_{L}$ (which depends on the size $L$ ) close to but less than 1 , such that at $V / J=\delta_{L}, E_{(\pi, \pi)}$ and $E_{(\pi, 0)}$ cross each other and $E_{(\pi, \pi)}$ becomes the first excited state for $\delta_{L}<V / J<1$. This crossing of energy levels may imply the existence of a new ground state in the region $\delta_{L}<V / J<1$. However, finite-size extrapolation analysis on $\delta_{L}$ shows that this is not the case. As shown in Fig. 3, $\delta_{L}$ approaches 1 as a power law of $L,{ }^{14}$

$$
\left(1-\delta_{L}\right) \propto L^{-2.13}
$$

In other words, such a spurious energy level crossing is only a finite-size effect. It does not occur in the thermodynamic limit and no transition is likely to occur at $V / J$ close to 1 . Therefore, we expect that the $E_{(\pi, 0)}$ state is always the lowest excited state in the range $-1 \leqslant V / J<1$. This is consistent with, but does not imply, the existence of long-range order (LRO) in the QDM at $-1 \leqslant V / J<1$.

Next we study the energy gap $\Delta E_{L}$, which is the energy difference between $E_{(\pi, 0)}$ and $E_{0}$. Figure 4 shows different plots of $\Delta E_{L}$ vs $L$ at different $V / J$. When $V / J$ is close to 1 , complication arises because of the spurious crossing over of the states $E_{(\pi, 0)}$ and $E_{(\pi, \pi)}$ at $\delta_{L}$. But we find that $\Delta E_{L}$ is not very sensitive to $V / J$ in the region close to $\delta_{L}$ (see Fig. 2). Consequently we plot $\Delta E_{L}$ at $V / J=\delta_{L}$ (instead of at the 


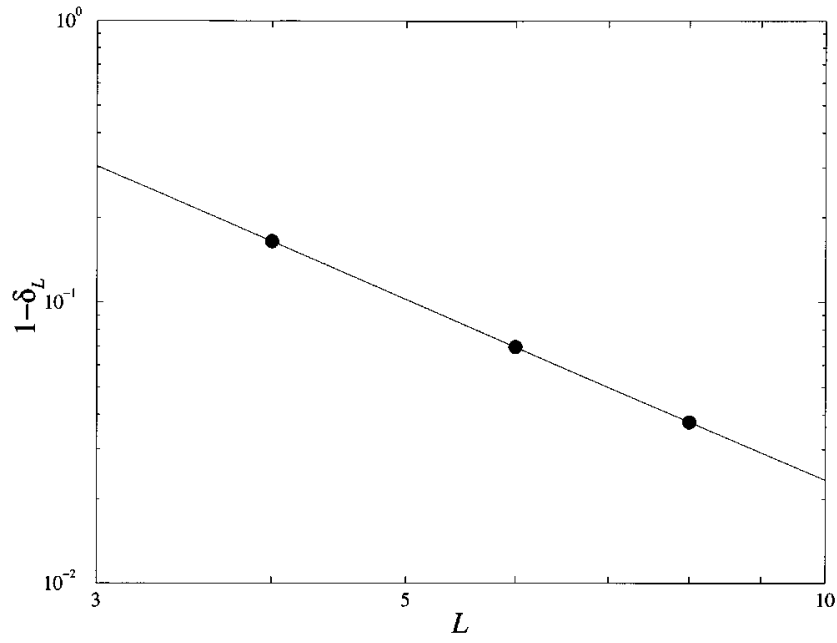

FIG. 3. $\left(1-\delta_{L}\right)$ vs $L$. The straight line is the best fit to the data, $1-\delta_{L}=3.1544 L^{-2.13}$.

same $V / J$ for different $L$ ) when $V / J$ is close to 1 . From Fig. 4 we can clearly distinguish two different behaviors. At $V / J=-1, \Delta E_{L}$ decays exponentially with $L$. This is a clear signal for the existence of LRO. When $0 \leqslant V / J<1$, we find that $\Delta E_{L}$ vanishes with a power law in $L$. Best fit to the data shows that the power is approximately 2 in the whole range. For $-1<V / J<0$, we are not able to determine whether $\Delta E_{L}$ has exponential or power law dependence on $L$. This is probably because of the more serious finite-size effect when $V / J$ is close to a transition point. Hence we infer that there are two phases in the range $-1 \leqslant V / J<1$. At some negative $V / J$, a transition occurs and the dependence of $\Delta E_{L}$ on $L$ changes from exponential to power law.
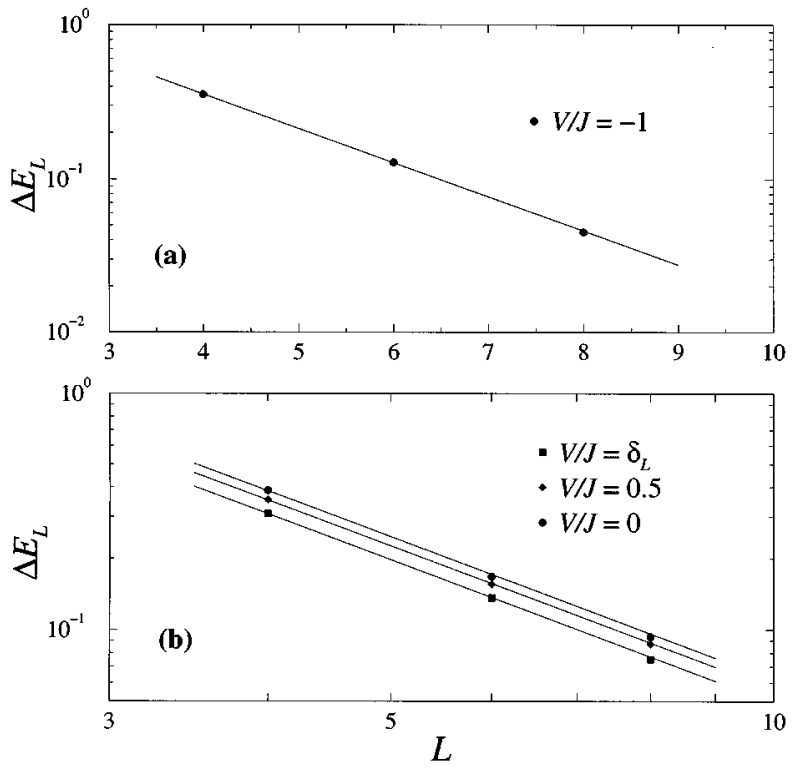

FIG. 4. (a) $\Delta E_{L}$ vs $L$ in semilogarithmic scale. The straight line is the best fit to the data, $\Delta E_{L}=2.742 e^{-0.511 L}$. (b) Similar plot but in logarithmic scale. $\delta_{L}$ is close to 1 (see Fig. 3). The straight lines are best fits to the data of the form $\Delta E_{L} \propto 1 / L^{2}$.

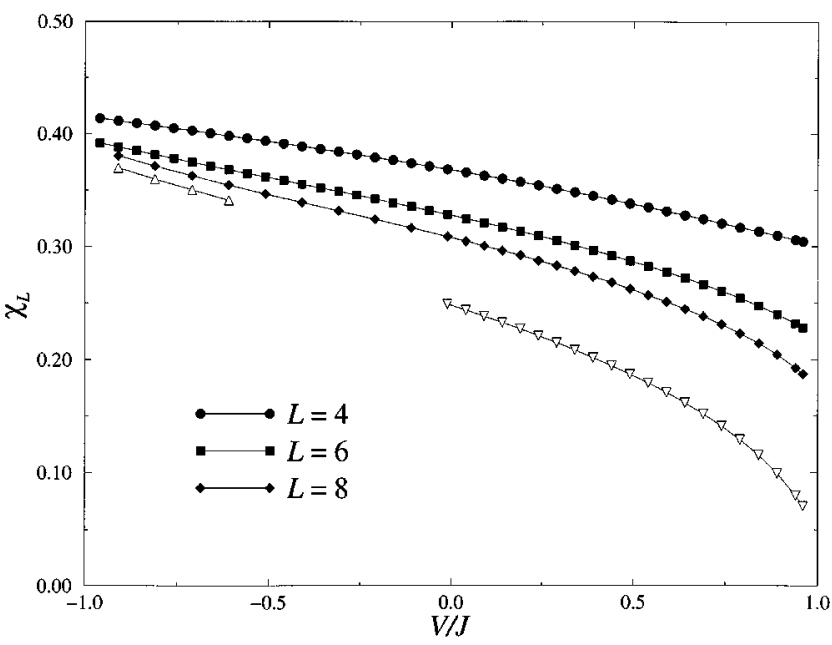

FIG. 5. $\chi_{L}$ vs $V / J$ for $L=4,6,8 . \triangle$ and $\nabla$ are $\chi_{\infty}$ obtained by extrapolating $\chi_{L}$ linearly in $1 / L^{2}$ and $1 / L$, respectively.

\section{COLUMNAR ORDER PARAMETER}

The columnar dimer order parameter is defined as ${ }^{8}$

$$
\begin{aligned}
\Psi_{\mathrm{col}}(\mathbf{r})= & (-1)^{r_{x}}\left[n\left(\mathbf{r}+\frac{\hat{\mathbf{x}}}{2}\right)-n\left(\mathbf{r}-\frac{\hat{\mathbf{x}}}{2}\right)\right] \\
& +i(-1)^{r_{y}}\left[n\left(\mathbf{r}+\frac{\hat{\mathbf{y}}}{2}\right)-n\left(\mathbf{r}-\frac{\hat{\mathbf{y}}}{2}\right)\right],
\end{aligned}
$$

where $\hat{\mathbf{x}}$ and $\hat{\mathbf{y}}$ are unit vectors. The dimer number operator $n(\mathbf{r}+\hat{\mathbf{e}} / 2)$ is 1 if the site at $\mathbf{r}$ and its nearest neighbor at $\mathbf{r}+\hat{\mathbf{e}}$ form a dimer, and zero otherwise. In the finite-size study, one defines the order parameter ${ }^{8}$

$$
\chi_{L}^{2}=\left\langle\left|\frac{1}{L^{2}} \sum_{\mathbf{r} \in A} \Psi_{\text {col }}(\mathbf{r})\right|^{2}\right\rangle .
$$

For a dimer liquid which has no long-range dimer order, $\chi_{L}$ is zero in the large $L$ limit. If long-range columnar order exists, $\chi_{L}$ remains finite at large $L$. In Fig. 5 we plot $\chi_{L}$ versus $V / J$. When $V / J$ is close to $-1, \chi_{L}$ is approximately linear in $1 / L^{2}$. But when $V / J$ is increased, a linear relation in $1 / L$ fits the data better. Unfortunately we are not able to determine the point where these two behaviors change from one to another. Using these linear relations, we extrapolate $\chi_{L}$ to obtain $\chi_{\infty}$, which is also plotted in Fig. 5. It shows that $\chi_{\infty}$ is finite at $V / J<1$, which signifies the existence of columnar order. $\chi_{\infty}$ decreases as $V / J$ approaches 1 , showing that the columnar order is weakening. But there is no evidence that $\chi_{\infty}$ is zero at any $V / J<1 . \chi_{\infty}$ clearly shows that the columnar order persists for $V / J<1$.

Different dependence of $\chi_{L}$ on $L\left(\propto 1 / L^{2}\right.$ and $\left.1 / L\right)$ may imply different orders in the respective regions. One defines the correlation function of $\Psi_{\mathrm{col}},{ }^{8}$

$$
G(\mathbf{r})=\left\langle\Psi_{\text {col }}^{*}\left(\mathbf{r}_{1}\right) \Psi_{\text {col }}\left(\mathbf{r}_{1}+\mathbf{r}\right)\right\rangle,
$$

where $\mathbf{r}, \mathbf{r}_{1}$ are in the $A$ sublattice. In the two regions where $\chi_{L}$ has finite-size corrections $1 / L^{2}$ and $1 / L, G(r)$ probably decays exponentially and as a power law in $r$, respectively, to a nonzero constant value. Figure 6 shows $G(r)$ in the $L=8$ system. Unfortunately, direct observation of how 


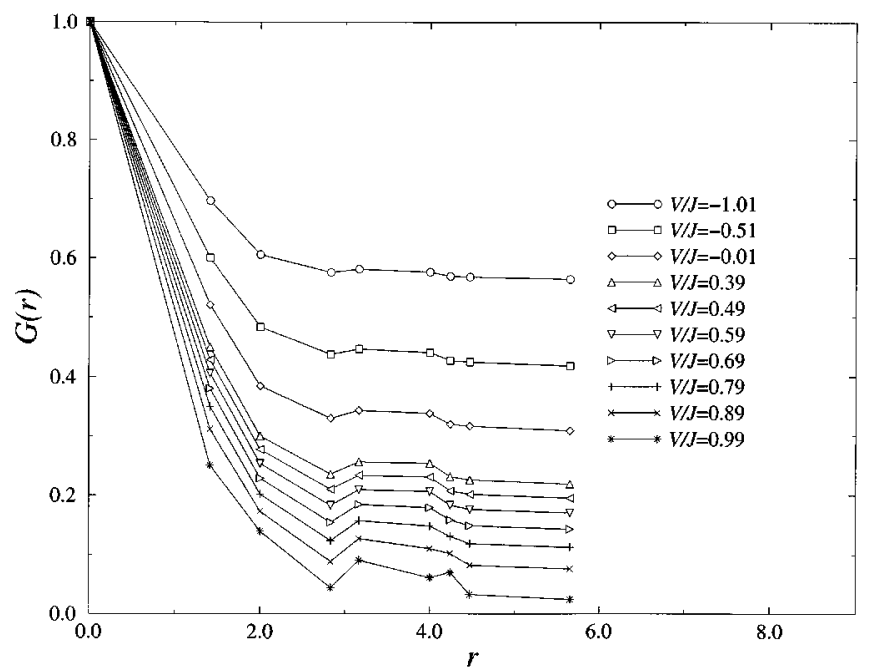

FIG. 6. $G(r)$ at different $V / J$ for $L=8$.

$G(r)$ decays with $r$ is not possible in this system size. Figure 6 shows that when $V / J$ is away from $1, G(r)$ is a nonzero constant at large $r$. When $V / J$ is close to 1 , it is not possible to tell from Fig. 6 whether $G(r)$ is small or zero at large $r$. Nevertheless, it tells us that in the range $-1 \leqslant V / J<1$ the long-range order, if exists, should be columnar.

As suggested in Ref. 8, a further probe of the columnar dimer order is provided by the cumulant of the columnar order parameter,

$$
g_{L}=\left\langle\left|\sum_{\mathbf{r}} \Psi_{\mathrm{col}}(\mathbf{r})\right|^{4}\right\rangle /\left\langle\left|\sum_{\mathbf{r}} \Psi_{\mathrm{col}}(\mathbf{r})\right|^{2}\right\rangle^{2} .
$$

If long-range columnar dimer order exists, $g_{L} \rightarrow 1$ as $L \rightarrow \infty$. We plot $g_{L}$ versus $V / J$ in Fig. 7(a). Standard finitesize scaling theory ${ }^{15}$ shows that at phase transition, $g_{L}$ at different $L$ cross at a unique point. Since $g_{L}$ do not cross except when $V / J$ is close to 1 , Fig. 7(a) tells us that the order parameter $\Psi_{\text {col }}$ detects no transition except when $V / J$ is close to 1 . This is the transition to the dimer liquid (FS) state. Since our system size $L$ is not large enough to make $g_{L}$ cross at one point, it is not possible to determine the transition point from Fig. 7(a). Nevertheless, our previous finite-size analysis of $\Delta E_{L}$ and $\chi_{L}$ strongly suggest that some kind of columnar LRO persists all the way up to $V / J=1$. We also note that $g_{L}$ detects no transition at any $-1 \leqslant V / J<0$, in contrary to the results of $\Delta E_{L}$ and $\chi_{L}$.

\section{DISORDER WITHIN THE COLUMNS}

It is easy to understand why $g_{L}$ is not able to detect the transition at $V / J<0$. Finite $\chi_{\infty}$ does not necessarily imply the kind of columnar order shown in Fig. 1(a). Take the plaquette RVB state as an example, $\chi_{L}$ is also nonzero in the large $L$ limit. It can be considered as a state which possesses long-range "columnar order" (as measured by $\chi_{L}$ ), but has disorder within the established columns. To detect this disorder we introduce an order parameter $M_{\| \text {, }}$,

$$
M_{\|,=}=\frac{1}{L^{2}} \sum_{\text {plaquettes }}[n(|||\rangle)-n(|=\rangle)],
$$

where $n(||\rangle)$ and $n(|=\rangle)$ are number operators of vertical $(\|)$ and horizontal $(=)$ dimer pairs, respectively. In the perfect columnar state, $\left|M_{\|,=}\right|=1 / 2$, while in the plaquette $\mathrm{RVB}$, dimer liquid and the staggered state, $M_{\|,=}=0$. Analogous to $\Psi_{\text {col }}$, we define the cumulant

$$
g_{\|,=}=\frac{\left\langle\left|M_{\|,=}\right|^{4}\right\rangle}{\left\langle\left|M_{\|,=}\right|^{2}\right\rangle^{2}} .
$$

Again, finite-size scaling theory shows that at the phase transition, $g_{\|,=}$at different $L$ should intersect at a unique point. In Fig. 7(b) we plot $g_{\|,=}$vs $V / J$ for different $L$. Although the three curves do not intersect at a single point, the crossing of the curves at $L=6$ and 8 indicates that a transition is possible at some negative value of $V / J$. This is consistent with the results of $\Delta E_{L}$ and $\chi_{L}$. We are not able to obtain a good estimation for the transition point $V_{c} / J$. But very roughly, $V_{c} / J \sim-0.2$.

We also calculate the moments of $M_{\mid,-}$,

$$
M_{\mid,-}=\frac{1}{L^{2}} \sum_{\text {plaquettes }}[n(\mid)-n(-)],
$$

i.e., the difference between the number of vertical and horizontal dimers. The corresponding cumulant $g_{\mid,-}$is shown in Fig. 7(c). We find a similar crossing of $g_{\mid,-}$for $L=6,8$ also near $V / J \sim-0.2$. We note that for $V>V_{c}$ both $g_{\|,=}$and $g_{\mid,-}$are close to 3 . This value is the expected Gaussian moment ratio for disordered states. This strongly suggests there is no $M_{\|,=}$or $M_{\mid,-}$LRO for the whole range of $V>V_{c}$. For $V / J<-1$ the cumulants approach 1 for the ordered columnar state. In the $L \rightarrow \infty$ limit the cumulants should become 1 for all $V<V_{c}$. Figures 7(b) and 7(c) are consistent with this scenario.

\section{DIMER CORRELATIONS}

$g_{\|,=}$and $g_{\mid,-}$show that the possible transition at $V_{c} / J$ is between two states with long-range columnar order, but one has disorder within the columns. Next we are going to study the dimer correlations in these two states. The spatial correlation of the dimers can be displayed by evaluating the dimer-dimer correlation function, ${ }^{16}$

$$
C_{(i j)(k l)}=\frac{\left\langle n_{i j} n_{k l}\right\rangle-\left\langle n_{i j}\right\rangle^{2}}{\left\langle n_{i j}^{2}\right\rangle-\left\langle n_{i j}\right\rangle^{2}},
$$

where $n_{i j} \equiv n\left[\frac{1}{2}\left(\mathbf{r}_{i}+\mathbf{r}_{j}\right)\right]$. If the dimers do not have LRO, $C_{(i j)(k l)}$ should show some short-range correlations, and fall off rapidly as the separation between the bonds $(i j)$ and $(\mathrm{kl})$ increases. On the other hand, if the dimers have LRO, $C_{(i j)(k l)}$ should reflect the pattern of the long-range correlations. Figure 8 shows the dimer-dimer correlation in the $L=8$ system at $V / J=-1$ and 0 . The reference bond $(i j)$ is represented by a double line. $C_{(i j)(k l)}$ is proportional to the thickness of the line joining the pair of sites $k$ and $l$. Solid line means $C_{(i j)(k l)}>0$ (correlation), and broken line means $C_{(i j)(k l)}<0$ (anticorrelation). It is obvious that in both cases the overall order is columnar-those bonds with positive $C_{(i j)(k l)}$ are arranged in well-defined columns. This is consistent with the study using $\Psi_{\text {col }}(\mathbf{r})$ as the order parameter (Sec. IV). Except for the trivial short-range correlations, 


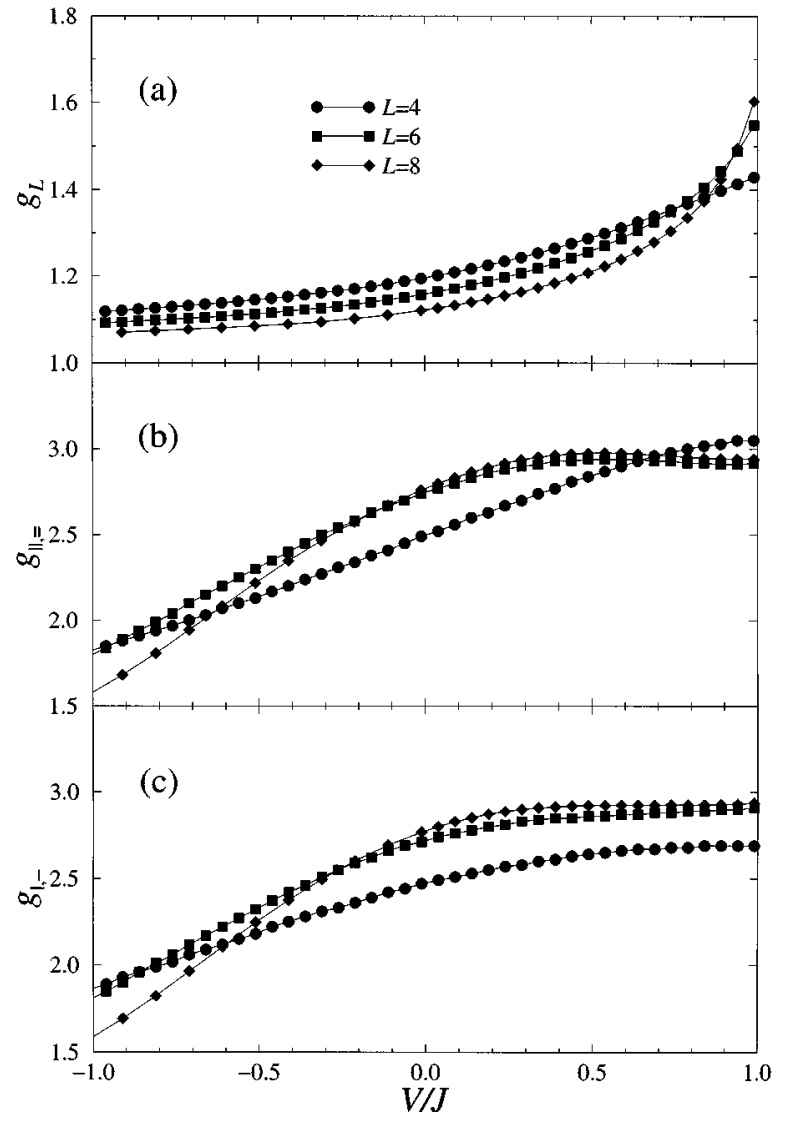

FIG. 7. Various cumulants vs $V / J$ for $L=4,6$, and 8. (a) $g_{L}$, (b) $g_{\|,=}$, and (c) $g_{\mid,-}$.

$C_{(i j)(k l)}$ does not fall off significantly with distance. This is an indication that the correlation is long range. A major difference between Figs. 8(a) and 8(b) is that in the latter, $C_{(i j)(k l)}$ for vertical $(k l)$ are very close to zero, except for those in contact with $(i j)$. In the perfect columnar state as shown in Fig. 1(a), $C_{(i j)(k l)}=1$ or $-1 / 3$ when the bond $(k l)$ forms and does not form dimer, respectively. [Here the reference bond $(i j)$ has been chosen to be one of the horizontal bonds that form dimer in Fig. 1(a).] This is to be compared to the result at $V / J=-1$ [Fig. 8(a)]. In the perfect plaquette RVB state as shown in Fig. 1(c), except for those $(k l)$ which are in the same plaquette as $(i j), C_{(i j)(k l)}=0$ for vertical $(k l)$, and $C_{(i j)(k l)}= \pm 1 / 3$ for horizontal $(k l)$, depending on whether $(k l)$ belongs to any one of the plaquettes shown in Fig. 1(c). In the same plaquette as $(i j)$, horizontal and vertical $(k l)$ have $C_{(i j)(k l)}=2 / 3$ and $-1 / 3$, respectively. This is to be compared to the result at $V / J=0$ [Fig. 8(b)]. Hence the dimer correlations strongly suggest that at $V / J=-1$, the ground state is ordered columns, whereas at $V / J=0$, it is consistent with the plaquette RVB state.

\section{PLAQUETTE RVB STATE AND THE TRANSITION AT $V_{c} / J$}

From the above results and discussions, there is evidence that at $V_{c} / J$, the ground state of the QDM changes from the ordered columnar to the plaquette RVB state. Since this transition does not appear to have been discussed in the literature, we will discuss it in a little more detail. One can moti-

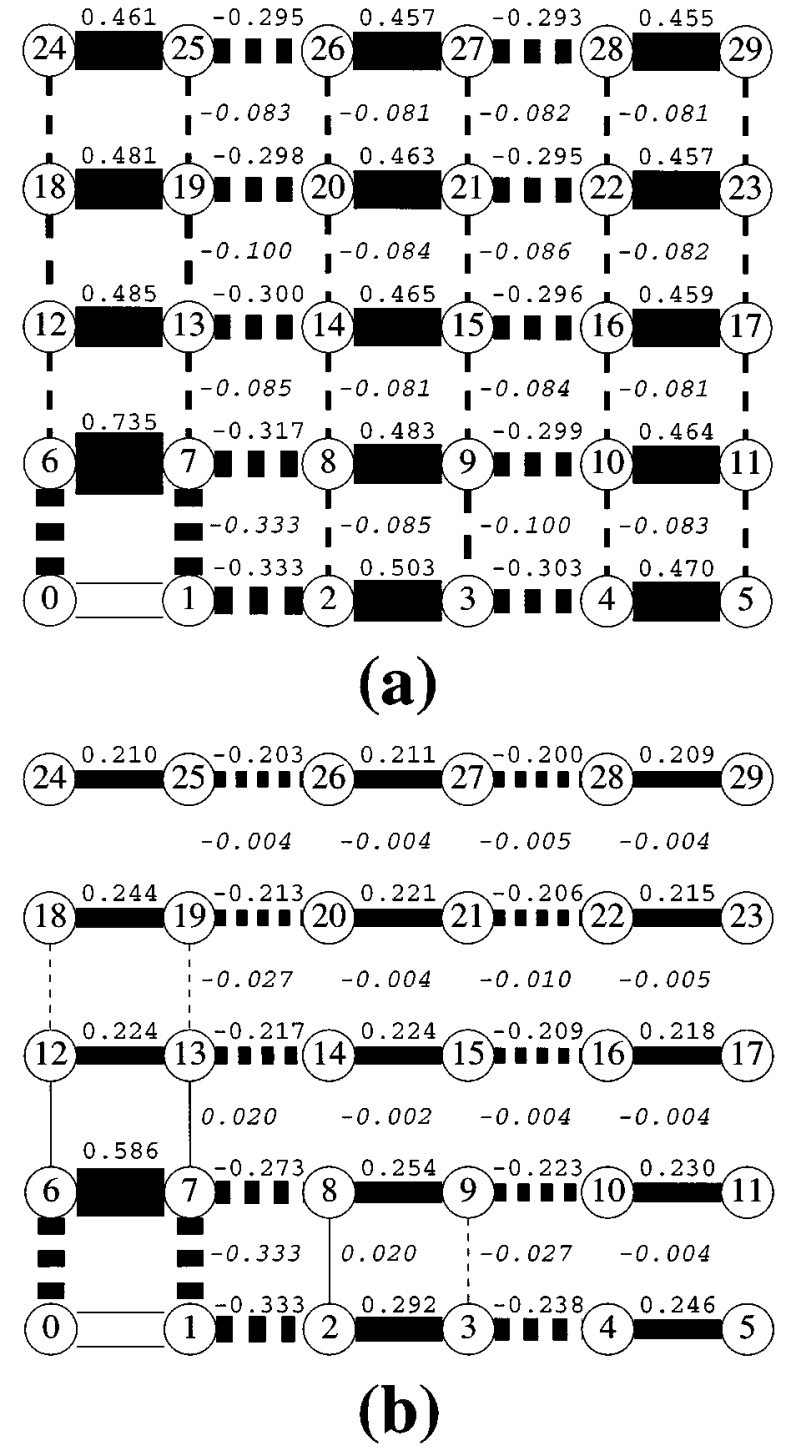

FIG. 8. Dimer-dimer correlation $C_{(i j)(k l)}$ in the $L=8$ system at (a) $V / J=-1$ and (b) $V / J=0$. Only a quadrant of the system containing all the inequivalent dimer pairs is shown. The reference bond $(i j)$ is represented by a double line. $C_{(i j)(k l)}$ is proportional to the thickness of the line joining the pair of sites $k$ and $l$. Solid line means $C_{(i j)(k l)}>0$, and broken line means $C_{(i j)(k l)}<0$. Note that the line widths are in the same scale in both cases. The values of $C_{(i j)(k l)}$ are shown next to the bonds, where different fonts are used for vertical and horizontal bonds.

vate the existence of this transition by a simple argument where the QDM is approximately mapped onto a twodimensional Ising model in a transverse field. The $T=0$ transition from ferromagnet to paramagnet in that model corresponds to the $V_{c} / J<0$ transition of the QDM discussed in the previous sections. We now outline the steps and implications of this approximate treatment.

First consider the case $V / J \ll-1$ where the system tends to the ordered columnar state in Fig. 1(a). The basic quantum fluctuations in the ground state are induced by the kinetic energy term in (1), namely by flipping $|=\rangle$ to $|\|\rangle$. For each dimer populated column in Fig. 1(a) consider every other plaquette [as in Fig. 1(c)] to have an index " $i$ ', and state variable $\sigma_{i}^{z}=1$ if it is in the state $|=\rangle$ and $\sigma_{i}^{z}=-1$ if it is 
$|\|\rangle$. Figure 1(a) has all $\sigma_{i}^{z}=1$, i.e., a ferromagnetic arrangement, whereas Fig. 1(c) has all $\sigma_{i}^{z}= \pm 1$ with probability $1 / 2$, i.e., a paramagnetic arrangement. Note also that the other ferromagnetic Ising configuration, with all $\sigma_{i}^{z}=-1$, gives another perfectly ordered columnar state. The collection of states generated by all possible $\left\{\sigma_{i}^{z}\right\}$ is, of course, a small subset of the set of all close-packed dimer configurations. However, they at least describe the plaquette RVB states, the ordered columnar states, and one-half of the basic flipping excitations. Given that our earlier numerical results suggest columnar order for all $V / J<1$, we do not worry too much that the $\left\{\sigma_{i}^{z}\right\}$ subset of dimer states are, by construction, constrained to have long-range columnar order, since we are concerned with the disordering of other degrees of freedom (e.g., dimer pair arrangements and correlations within each established column).

Limiting the system to the collection of all possible $\left\{\sigma_{i}^{z}\right\}$ values, one can show that the Hamiltonian of the QDM is, to within an additive constant,

$$
\mathcal{H}=-J \sum_{i} \sigma_{i}^{x}+\frac{V}{4} \sum_{\langle i j\rangle} \sigma_{i}^{z} \sigma_{j}^{z}
$$

where the sums are over every other plaquette as shown in Fig. 1(c). $\sigma_{i}^{x}$ and $\sigma_{i}^{z}$ are Pauli operators. The kinetic energy term with $J$ now induces flipping of the $\sigma^{z}$ variables (like a transverse Ising field $h_{x}$ ), and the potential energy term $V$ introduces interactions (like an Ising exchange integral $\mathcal{J}_{z}$ ) between neighboring plaquettes as shown in Fig. 1(c). ${ }^{17}$

For $V / J \ll-1$ all the $\sigma_{i}^{z}$ are arranged ferromagnetically, whereas for some $V_{c} / J<0$ the system undergoes a continuous phase transition (in the universality class of the classical 3D Ising model) to a state with no LRO in the $\sigma_{i}^{z}$ variables. Indeed, the case $V=0$ is trivially seen to be perfectly disordered with respect to the $\sigma_{i}^{z}$ variables, and is precisely the state represented schematically in Fig. 1(c). For $V_{c}<V<0$ there is some short ranged $\sigma^{z}$ order, but no LRO. Since our $(=)(\|)$ pairs of dimers are neither allowed to break apart nor to move to neighboring plaquettes, this approximation has long-range columnar order (as measured by $\Psi_{\text {col }}$ ) preserved past the transition point $V_{c}$. Our numerical data suggests this to be the case for the QDM as well: LRO in $\Psi_{\text {col }}$ exists for all $V / J<1$ but there is a loss of LRO in $M_{\|,=}$at some negative value of $V$.

\section{FINITE TEMPERATURE PHASE DIAGRAM}

Although our numerical calculations are limited to the $T=0$ ground state of the QDM, it is interesting to speculate on the behavior of the system at finite temperatures. The simplest, and we feel most likely, scenario is displayed schematically in Fig. 9. For any fixed ratio $V / J$, as $T \rightarrow \infty$ the only interaction that matters is the hard-core dimer constraint, and so the FS dimer liquid will always be obtained at high enough temperature. We expect that for fixed $V<V_{c}$ as $T$ is increased the ordered columnar state will give way to the same intermediate state identified above (i.e., columnar with disorder, and consistent with plaquette RVB order), and at a second higher temperature the columnar order will be destroyed as the system enters the high-temperature dimer

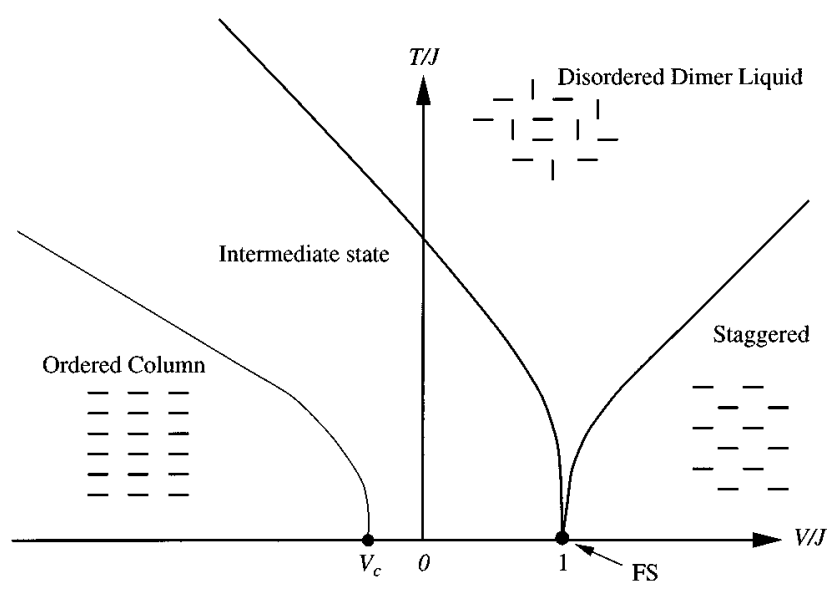

FIG. 9. Speculated phase diagram of the QDM.

liquid phase. Similarly, for $V>J$ the staggered phase in Fig. 1(b) will be destroyed at high enough $T$. The simplest guess is that it goes directly to the dimer liquid, ${ }^{18}$ although it is not possible to rule out other phases. It seems likely that the high temperature dimer liquid region makes it all the way down to the $T=0$ axis, but only at the point $V=J$. It is intriguing that the $T=\infty$ Fisher-Stephenson (FS) state is exactly regained at $T=0$ at a single point.

\section{CONCLUSION}

To conclude, we find no evidence of a dimer liquid state in the QDM in the range $-1 \leqslant V / J<1$. In this range, the QDM exists in two states. At $-1 \leqslant V / J<V_{c} / J$, the ground state possesses long-range columnar dimer order as suggested in Fig. 1(a). Its columnar order correlation function $\left\langle\Psi_{\text {col }}(0) \Psi_{\text {col }}(\mathbf{r})\right\rangle$ is likely to decay to a constant value exponentially with $r$, and it has exponentially vanishing energy gap. This state should exist at any $V / J \leqslant-1$, because negative $V$ favors parallel dimers. At $V_{c} / J<V / J<1$, the ground state possesses columnar order (as measured by $\chi_{L}$ ), but has disorder within the established columns. Its columnar order correlation $\left\langle\Psi_{\text {col }}(0) \Psi_{\text {col }}(\mathbf{r})\right\rangle$ is likely to decay to a constant value as a power law of $r$, and the energy gap vanishes as a power of $L$. The symmetries of the low lying excited states and the spatial dimer correlations of the ground state are consistent with plaquette RVB order as illustrated in Fig. 1(c). By approximately mapping the QDM to an Ising model with a transverse field, we argue for the existence of a transition at $V_{c}$. Unfortunately, our system sizes do not allow us to determine the precise value of $V_{c} / J$. Our rough estimate is $V_{c} / J \sim-0.2$. We note that we cannot rule out additional transitions in the range $V_{c} / J<V / J<1$, we can only say the quantities we have measured do not indicate any more.

Compared to earlier work ${ }^{8}$ which concluded that a dimer liquid state may exist in $\kappa \leqslant V / J \leqslant 1$ and gave an upper bound of 0.5 to $\kappa$, we are able to perform more reliable finite-size scaling analysis with the addition of the $L=8$ results. Our results push $\kappa$ to very close to 1 which suggest that $\kappa$ is in fact 1 . Finally, we remark that in the quantum mechanical height model, the corresponding transition at $V_{c}$ is predicted to be first order. ${ }^{13}$ Unfortunately the system sizes 
we can deal with are too small to determine $V_{c}$ accurately, let alone to estimate the critical exponents.

\section{ACKNOWLEDGMENTS}

P.W.L. thanks C. Zeng for very useful discussions, and C. L. Henley for communicating his results before publication. This work was supported in part by Hong Kong RGC Grant No. HKUST619/95P.

\section{APPENDIX A: WINDING NUMBERS}

Following Ref. 1 we use the columnar state as the reference configuration to define the winding numbers. In Fig. 10 (a), the dimers of such a reference configuration are indicated by dash lines. The arrows along the dimers always point from the $A$ sublattice (solid circles) to the $B$ sublattice (open circles). In Fig. 10(b), a dimer configuration (indicated by solid lines) is superimposed on the reference configuration, but the arrows along the dimers (solid lines) point from the $B$ sublattice to the $A$ sublattice. This results in directed close paths which may wrap around the boundaries (with periodic boundary conditions). The winding numbers $\Omega_{x}$ and $\Omega_{y}$ are the net number of loops (clockwise minus counterclockwise) wrapping around the boundaries in the $x$ and $y$ directions, respectively. Each dimer configuration has a unique pair of winding numbers. By repeatedly flipping parallel dimer pairs (i.e., applying $\mathcal{H}$ ) of a dimer configuration, one can generate all dimer configurations with the same winding numbers. But no two configurations with different winding numbers are related by such dimer flipping operations. Therefore, the Hilbert space spanned by all closepacked dimers can be divided into subspaces labeled by winding numbers.

To calculate the winding numbers of a given dimer configuration, we start from a site and traverse the lattice following the arrows as described above until we return to the starting site. We mark all the sites along this path and count the net number of times it crosses the boundaries in the $x$ and $y$ directions. This procedure is repeated starting from an unmarked site until all sites are marked. It is easy to see that the columnar dimer state in Fig. 1(a) has winding numbers $(0,0)$, and the staggered state in Fig. 1(b) has winding numbers $( \pm L / 2,0)$.

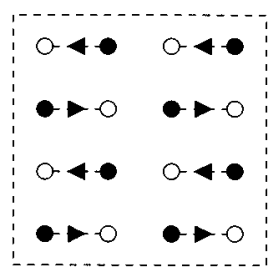

(a)

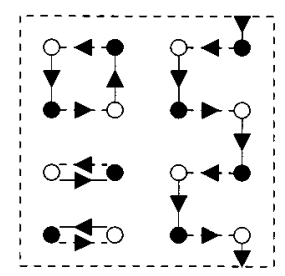

(b)
FIG. 10. (a) The reference columnar dimer configuration, with arrows pointing along the dimers from the $A$ sublattice $(\bullet)$ to the $B$ sublattice $(\bigcirc)$. (b) A dimer configuration (indicated by solid lines) superimposed on (a), but with arrows pointing in the opposite direction, i.e., from the $B$ sublattice $(\bigcirc)$ to the $A$ sublattice $(\bigcirc)$. This dimer configuration has winding numbers $(0,1)$ or $(0,-1)$.

\section{APPENDIX B: ENUMERATING DIMER CONFIGURATIONS}

In this Appendix we describe the way we generate all the close-packed dimer configurations on an $L \times L$ lattice. Again we divide the square lattice into two sublattices $A$ and $B$. At close pack, each site is one end of exactly one dimer. Consequently, a dimer configuration can be represented by specifying the direction $( \pm \hat{\mathbf{x}}$ or $\pm \hat{\mathbf{y}}$ ) of the dimer attached to each site in the $A$ sublattice. There are $2^{L^{2}}$ such combinations. But most of them are forbidden because some sites in the $B$ sublattice have zero or more than one dimer attached. The most straightforward way to enumerate all the dimer configurations is to scan through these $2^{L^{2}}$ combinations to identify the allowed configurations. But this takes prohibitively long for $L=8$. We need a clever way to identify and eliminate the forbidden configurations quickly.

We further divide the $A$ sublattice into two quarterlattices $A_{0}$ and $A_{1}$. Each site takes up two bits in a computer word, with the four binary values $00,01,10$, and 11 representing the direction of the dimer attached to it. We divide our task into two steps. First we generate allowed configurations for $A_{0}$ alone, without considering $A_{1}$. Then we generate the allowed configurations for $A_{1}$ under the constraints of the $A_{0}$ configurations. The $A_{0}$ and $A_{1}$ configurations together give all the allowed dimer configurations.

$A_{0}$ has $L^{2} / 4$ sites. Each $A_{0}$ configuration is represented in the computer by an integer word with at least $2 \times\left(L^{2} / 4\right)=L^{2} / 2$ bits. The $2 j$ th and $2 j+1$ th bits of the integer word represent the direction of the dimer attached to the $j$ th site of $A_{0}$. In this way each $A_{0}$ configuration is uniquely associated with an integer value. To scan through all the possible $A_{0}$ configurations, we start from an integer value $2^{L^{2} / 2}-1$ whose binary representation has 1 's in the lower $L^{2} / 2$ bits, and 0 's otherwise. This integer value corresponds to the $A_{0}$ configuration with all dimers pointing in the same direction. Successively decreasing this integer value by 1 is equivalent to rotating the dimers in all possible directions starting from the 0 th, 1 st, ... sites. In this way all possible $A_{0}$ configurations can be generated. For each integer value (i.e., possible $A_{0}$ configuration), we check for conflict starting from the last site. If at some stage we find that the dimers attached to the $i$ th and $j$ th sites $(i>j)$ of $A_{0}$ are conflicting (both connect to the same site in the $B$ sublattice), we can skip all the bits lower than the $2 j$ th bit, i.e., instead of decrementing the integer value by 1 , we can go to the next possible configuration by changing the $2 j$ th and $2 j+1$ th bits. This substantially decreases the run time. For $L=8$, $A_{0}$ has 9983558 allowed configurations.

The set of allowed configurations for $A_{1}$ is the same as that for $A_{0}$. Naively we can match these two sets of configurations and eliminate the forbidden ones. But this involves $(9983558)^{2} \sim 10^{14}$ steps for $L=8$ and will take prohibitively long. We must fully utilize the geometrical constraints imposed by the $A_{0}$ configurations when enumerating the possible $A_{1}$ configurations. For a particular $A_{0}$ configuration, we mark the allowed dimer directions for each site in $A_{1}$ under the constraints imposed by that $A_{0}$ configuration. The total number of combinations is substantially smaller than $2^{L^{2} / 2}$. 
We then scan through all these combinations in the same manner as in enumerating the $A_{0}$ configurations. Repeating the same procedure generates all the allowed dimer configurations. For $L=4,6$, and 8 , the number of allowed dimer configurations are 272, 90176 , and 311853 312, respectively. For $L=8$, all the allowed dimer configurations can be enumerated in about four hours using an HP 735 workstation.
*Electronic address: phleung@usthk.ust.hk

${ }^{1}$ D. Rokhsar and S. Kivelson, Phys. Rev. Lett. 61, 2376 (1988).

${ }^{2}$ N. Read and S. Sachdev, Nucl. Phys. B316, 609 (1989).

${ }^{3}$ N. Read and S. Sachdev, Phys. Rev. Lett. 62, 1694 (1989); S. Sachdev and N. Read, Int. J. Mod. Phys. B 5, 219 (1991).

${ }^{4}$ M. P. Gelfand, R. R. P. Singh, and D. A. Huse, Phys. Rev. B 40, 10801 (1989).

${ }^{5}$ P. W. Leung and N. Lam, Phys. Rev. B 53, 2213 (1996).

${ }^{6}$ C. Zeng and V. Elser, Phys. Rev. B 51, 8318 (1995).

${ }^{7}$ M. E. Fisher and J. Stephenson, Phys. Rev. 132, 1411 (1963).

${ }^{8}$ S. Sachdev, Phys. Rev. B 40, 5204 (1989).

${ }^{9}$ L. S. Levitov, Phys. Rev. Lett. 64, 92 (1990).

${ }^{10}$ P. Orland, Phys. Rev. B 49, 3423 (1994).

${ }^{11}$ T. Dombre and G. Kotliar, Phys. Rev. B 39, 855 (1989).

${ }^{12}$ M. E. Zhitomirsky and K. Ueda (unpublished).

${ }^{13}$ S. Sachdev and N. Read (unpublished).
${ }^{14}$ This power law behavior has been predicted using a "height analysis" of the QDM, C. L. Henley (private communications). See also C. L. Henley (unpublished).

${ }^{15} \mathrm{~K}$. Binder, in Finite Size Scaling and Numerical Simulation of Statistical Systems, edited by V. Privman (World Scientific, Singapore, 1990).

${ }^{16}$ P. W. Leung and V. Elser, Phys. Rev. B 47, 5459 (1993).

${ }^{17}$ We note that if the "expansion"' is performed about staggered columns (every other dimer column shifted by one plaquette) additional terms proportional to $V \sum \sigma_{i}^{z}$ will appear in $\mathcal{H}$, but this does not change our conclusions that a disordering transition occurs for some $V / J<0$.

${ }^{18}$ The substantial difference in symmetry and winding numbers between the staggered phase and the dimer liquid may mean that the transition between them is first order. 